LETTER TO THE EDITOR

\title{
ON THE VALIDITY OF THE WACHSTEIN AND MEISEL METHOD FOR ADENOSINE TRIPHOSPHATASE
}

Rosenthal et al. (J. Histochem. Cytochem. 14: 698. 1966) and Moses et al. (J. Histochem. Cytochem. $14: 702.1966$ ) have recently found that lead ions are capable of catalyzing the nonenzymatic hydrolysis of nucleoside phosphate and that the amount of inorganic phosphate precipitated in fixed tissue sections following incubation in the Wachstein and Meisel (WM) medium is in the same order of magnitude as the inorganic phosphate released by the lead-catalyzed hydrolysis of ATP in the medium. These findings naturally arose questions concerning the validity of the method. In defense of the WM method, Dr. Novikoff (J. Histochem. Cytochem. $15: 353,1967$ ) has already presented several data. However, Moses and Rosenthal (J. Histochem. Cytochem. 15: 354. 1967) considered that the experiments from which these data were derived have not been adequately controlled and that the data do not answer appropriately to the question of the validity of the WM method. In this connection, it seems worthy of discussing our published and unpublished data concerning the ATPase staining in rat liver and kidney by the WM method, taking into consideration the possibility of nonenzymatic hydrolysis of ATP by lead.

The reactions obtained in frozen-substituted sections by means of the WM method (ATP as substrate) can be classified roughly into six on the basis of their substrate specificity, sensitivities to inhibitors and fixatives, and requirement for bivalent cations (Hori and Chang, J. Histochem. Cytochem. $\left.11: 71.1963^{1}\right)$ : (1) the reaction demonstrated only in the presence of $\mathrm{Mg}$ and inhibited by $\mathrm{Ca}$, formalin, ethanol, $\mathrm{ADP}$, azide and $\mathrm{PCMB}$, but not by AMP, cyanide, and fluoride. The PCMB inhibition is reversed by glutathione. This is found in the cytoplasm of hepatic cells and of proximal convolutions of the kidney, and appears to be due to the ATPase, E.C. 3.6.1.4. (2) The reactions being same as the above except that $\mathrm{Ca}$ is effective in stimulating the reaction. This is found in muscles and appears to be partly due to the ATPase, E. C. 3.6.1.3. (3) The reaction demonstrated even in the absence of bivalent cations, stimulated by $\mathrm{Mg}$ and $\mathrm{Ca}$, resistant considerably to PCMB, azide, formalin, ADP, cyanide, and fluoride, and inhibited by ethanol completely. This is found in ascending limbs and distal convolutions of the kidney. The enzyme responsible for this reaction has been tentatively named the ATPase-like enzyme. (4) The reaction demonstrated with ADP as well as ATP as substrate in the presence of either $\mathrm{Mg}$ or $\mathrm{Ca}$, resistant

1) The amount of $2 \%$ lead nitrate described in the paper should read $0.6 \mathrm{ml}$, to give a final concentration of $3.6 \mathrm{~m} M$ for lead. 
to formalin, PCMB and azide, and inhibited by ethanol. Fluoride is inhibitory only to the reaction with ADP. This is found in bile canal, where the presence of more than one enzyme responsible for ATP and ADP dephosphorylations has been suggested. (5) The reaction demonstrated with ADP as well as ATP as substrate in the absence of bivalent cations, stimulated by $\mathrm{Mg}$ or $\mathrm{Ca}$, resistant to $\mathrm{PCMB}$, azide, cyanide, fluoride and formalin, and inhibited by ethanol. This is found in blood vessel walls. (6) The reaction demonstrated with either ATP, ADP, AMP, or glycerophosphate as substrate, resistant to ethanol and formalin, and inhibited by cyanide. This is found in brush borders of the proximal convolutions. The reactions 4-6 are excluded from the objectives of the present discussion, since these can not be regarded as a result of the action of true ATPases or a single enzyme.

It appears less likely that the nonenzymatic hydrolysis of ATP by lead is responsible for reaction 1 , since the reaction was completely abolished by the addition of ADP (similarly hydrolysable with lead ions, Rosenthal et al J. Histochem. Cytochem. $14: 698$. 1966) to the ATP containing medium, and also by replacement of $\mathrm{Mg}$ by $\mathrm{Ca}$ (known to catalyze ATP hydrolysis and transphosphorylation). Likewise, reaction 2 can not be accounted for on the basis of the nonenzymatic hydrolysis of ATP, since the nature of the reaction accords well with available biochemical bata on myofibrillar ATPase. In addition, muscles does not stain with ADP as substrate ${ }^{2}$, but it stains when creatine phosphate, a well-known unstable phosphate easily hydrolyzed nonenzymatically, is used as substrate in addition to ADP (Hori, Histochemie 7 : 297. 1966). In this case, the combined action of the creatine kinase and ATPase, but not the nonenzymatic hydrolysis of ADP appears to be responsible for the reaction. On the other hand, reaction 3 can not be ascribed to any of the ATPases which have been reported by biochemists. Only reason we regard this reaction as due to the ATPase-like enzyme is its substrate specificity; the reaction is obtained with ATP, but not with ADP. Peculiarity of this reaction was also demonstrated with the WM medium in which $\mathrm{pH}$ was lowered to 6 , and with the medium in which lead concentration was reduced to $0.6 \mathrm{mM}$. In either case, the cytoplasm of tubular epithelium of ascending limbs and of distal convolutions is stained, while the cytoplasm of hepatic cells and of the primary convolutions was not stained with the above media. If the nonenzymaitc hydrolysis of ATP would account for some positive reactions obtained by the WM method, reaction 3 might be an example of it. However, data are still insufficient to conclude that this is the case.

An important result obtained with the WM medium in which lead concentration was reduced to $0.6 \mathrm{~m} M$ is the staining of nucleolus or nucleolusassociated chromatin. Such staining decreased with increasing concentrations of lead and was not observed when lead concentration was above 3.0 $\mathrm{m} M$. We have carried out several experiments in order to ascertain whether

2) This observation was made with $10 \mu$ sections. If thicker sections were made, a weak staining was noted at the I band level. Under an electron microscope, the reaction product was found in triads. 


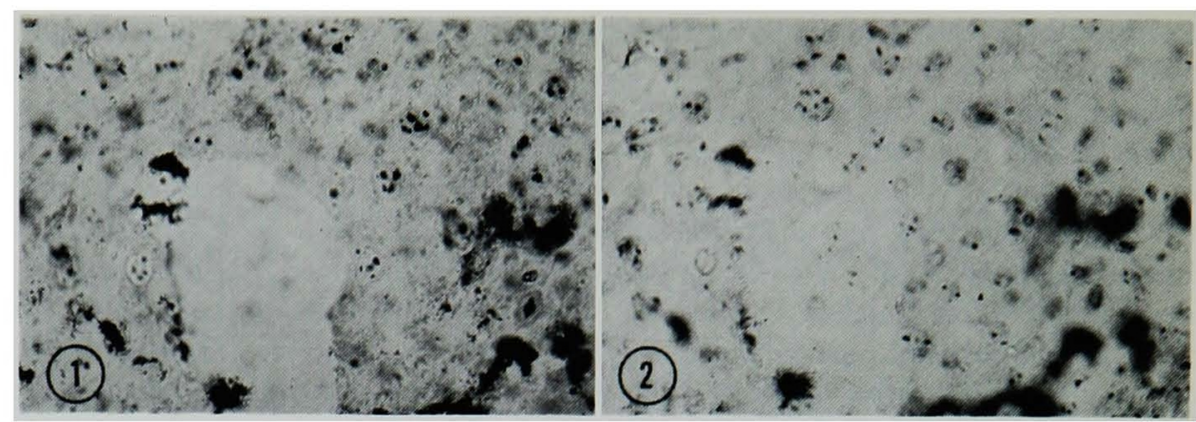

FIGS. 1 and 2. Staining of an active section (Fig. 1) superimposed on a heatinactivated section (Fig. 2) with the Wachstein-Meisel medium in which lead was $1 / 6$ of the original concentration. In both sections nucleoli or nucleolusassociated chromatin were stained. $\times 400$.

the staining of chromatin or nucleoli is due to a lead-sensitive enzyme or diffusion artifact or nonenzymatic transphosphorylation. One example of such experiments is illustrated in Figures 1 and 2, where an active section was superimposed on a heat-inactivated section and incubated in the low-lead medium. In both sections, nucleoli or nucleolus-associated chromatin were stained. On the contrary, such staining was not observed when lead concentration was $3.6 \mathrm{mM}$. This seems to indicate that the staining seen in the active section is due to the diffusion of the reaction product. However, the results of our other experiments disproved the possibility that the nucleolus or chromatin has an affinity to lead, lead phosphate, phosphate, or phosphatase, thus suggesting the complexity of mechanism involved in the staining of these nuclear elements at low lead concentrations.

In spite of the lack of our knowledge on the exact mechanism of the phosphatase staining with the lead method, it seems to be reasonable to assume that the WM method for ATPase would yield reliable results at least when the method is applied to unfixed, or only briefly fixed frozen sections.

Samuel H. Hori

Zoological Institute

Hokkaido University

Sapporo, Japan 